\title{
DINÁMICA DE LA CONCENTRACIÓN DE CALCIO SANGUÍNEO DURANTE EL PERIPARTO Y SU RELACIÓN CON PRODUCCIÓN Y REPRODUCCIÓN EN UN HATO DE VACAS JERSEY ${ }^{1}$
}

\author{
Alejandro Saborío-Montero ${ }^{2 / *}$, Jorge Ml. Sánche $z^{\dagger / *}$, María Vargas-Camacho* \\ Palabras clave: Hipocalcemia subclínica; hipocalcemia clínica; vacas lecheras en pastoreo. \\ Keywords: Subclinical hypocalcemia; clinical hypocalcemia; grazing dairy cows.
}

Recibido: 20/04/17

RESUMEN

El objetivo de esta investigación fue describir la dinámica de la concentración de calcio en suero sanguíneo durante el periparto en un hato de vacas Jersey de Oreamuno, Cartago, Costa Rica y su relación con indicadores productivos y reproductivos. Un total de 161 vacas fueron muestreadas, su dieta preparto consistió en pastoreo de kikuyo (Kikuyuocloa clandestina) (15,6\% de PC, 1,65 Mcal de $\mathrm{EN}_{\mathrm{L}}(3 \mathrm{X}), 0,35 \%$ de $\mathrm{Ca}, 0,32 \%$ de $\mathrm{Mg}, 3,5 \%$ de $\mathrm{K}$ ), suplementado con 4 kg.vaca ${ }^{-1} \cdot$ día $^{-1}$ de alimento balanceado bajo en calcio $(88 \% \mathrm{MS}, 14 \% \mathrm{PC}, 0,2 \% \mathrm{Ca}, 0,42 \%$ $\mathrm{Mg}$ y $1,38 \% \mathrm{~K})$, y $1 \mathrm{~kg}$ de heno $(82,3 \% \mathrm{MS}$, $5,1 \% \mathrm{PC}, 0,4 \% \mathrm{Ca}, 0,35 \% \mathrm{Mg}$ y $1,8 \% \mathrm{~K}) \cdot \mathrm{dia}^{-1}$. Inmediatamente después del parto, las vacas fueron suplementados con $1 \mathrm{~kg}$ de alimento balanceado (87,5\% MS, $18,6 \% \mathrm{PC}, 0,90 \% \mathrm{Ca}$, $0,42 \% \mathrm{Mg}$ y $1,38 \% \mathrm{~K}$ ) por cada $2,5 \mathrm{~kg}$ de leche. Se tomaron muestras de sangre de los vasos sanguíneos coccígeos desde 7 días preparto hasta 7 días posparto. Las concentraciones de $\mathrm{Ca}$ en las muestras de sangre fueron determinadas por espectrofotometría de absorción atómica. La concentración media mínima de calcio durante el estudio se obtuvo el día del parto $\left(7,27{\mathrm{mg} . \mathrm{dl}^{-1}}^{-1}\right)$

\footnotetext{
1 Trabajo financiado por la Vicerrectoría de Investigación. Proyecto 739-B5191. Universidad de Costa Rica.

2 Autor para correspondencia. Correo electrónico: alejandro.saboriomontero@ucr.ac.cr $\dagger 1951-2015$
}

Aceptado: 05/07/17
Dynamics of blood calciumconcentration
during peripartum and its relationship with
production and reproduction in a Jersey cow herd. The aim of this research was to describe the dynamics of calcium concentration in blood serum during peripartum in a Jersey cow herd from Oreamuno, Cartago, Costa Rica and its relationship with productive and reproductive indicators. A total of 161 cows were sampled, their pre-partum diet consisted grazing of kikuyu (Kikuyuocloa clandestina) $(15.6 \%$ CP, 1.65 Mcal NE $\left.\mathrm{L}_{\mathrm{L}}(3 \mathrm{X}), 0.35 \% \mathrm{Ca}, 0.32 \% \mathrm{Mg}, 3.5 \% \mathrm{~K}\right)$ supplemented with $4 \mathrm{~kg} \cdot \mathrm{cow}^{-1}$.day ${ }^{-1}$ of a low $\mathrm{Ca}$ balanced grain mix $(88 \% \mathrm{DM}, 14 \% \mathrm{CP}, 0.2 \% \mathrm{Ca}$, $0.42 \% \mathrm{Mg}$ and $1.38 \% \mathrm{~K})$, and $1 \mathrm{~kg}$ of hay $(82.3 \%$ $\mathrm{DM}, 5.1 \% \mathrm{CP}, 0.4 \% \mathrm{Ca}, 0.35 \% \mathrm{Mg}$ and $1.8 \%$ $\mathrm{K})$. day $^{-1}$. Immediately after calving, cows were supplemented with $1 \mathrm{~kg}$ of a balanced grain mix (87.5\% DM, $18.6 \% \mathrm{CP}, 0.90 \% \mathrm{Ca}, 0.42 \% \mathrm{Mg}$ and $1.38 \% \mathrm{~K}$ ) per each $2.5 \mathrm{~kg}$ of milk. Blood samples were taken from the coccygeal vessels since 7 days before calving until 7 days after calving. $\mathrm{Ca}$ concentrations in blood samples were determined by atomic absorption spectrophotometry. The average minimum concentration of $\mathrm{Ca}$ during the

\footnotetext{
Universidad de Costa Rica, Centro de Investigación en Nutrición Animal y Escuela de Zootecnia, Costa Rica.
} 
y en promedio las vacas superaron el umbral de hipocalcemia subclínica $\left(8,0 \mathrm{mg} \mathrm{dl}^{-1}\right)$ hasta el día 7 posparto. La concentración sanguínea de calcio no se asoció estadísticamente a indicadores reproductivos. Vacas multíparas con concentraciones de calcio inferiores a 5,5 mg. $\mathrm{dl}^{-1}$ el día del parto tuvieron estadísticamente $(\mathrm{p}<0,05)$ menor: producción por día, producción total y longitud de lactancia, en relación con vacas multíparas con concentraciones de calcio mayor o igual a 5,5 $\mathrm{mg} \cdot \mathrm{dl}^{-1}$. Estos hallazgos indican que la concentración de calcio sanguíneo durante el periparto es un factor asociado al desempeño productivo en vacas multíparas.

\section{INTRODUCCIÓN}

La hipocalcemia es un desbalance metabólico que afecta entre el 3 y 10\% de las vacas lecheras durante el periparto (Sánchez y Goff 2006); esta enfermedad se clasifica como clínica o subclínica en función de la concentración de calcio en suero sanguíneo. El valor umbral de concentración de calcio por debajo del cual se clasifican como vacas con hipocalcemia clínica es $5,5 \mathrm{mg}^{-\mathrm{dl}^{-1}}$, mientras que para las subclínicas es entre 5,5 y $8 \mathrm{mg}^{-\mathrm{dl}^{-1}}$ (Australia, State Goverment of Victoria 2007). El calcio es necesario para el normal funcionamiento del animal; sus principales funciones fisiológicas son la formación ósea, contracción muscular, trasmisión nerviosa, coagulación sanguínea y regulación de procesos hormonales (Horst et al. 1994). Se ha reportado que concentraciones deficientes de este mineral suprimen la respuesta inmune del animal (Kimura et al. 2006).

Durante el periparto el animal pasa de un estado de gestación, en el cual requiere alrededor de $12 \mathrm{~g}$ de $\mathrm{Ca}$ disponible por día para su mantenimiento y desarrollo fetal, a un estado en que sintetiza calostro con 1,7 a 2,3 g de Ca y posteriormente leche con 1,1 g de Ca por litro (NRC 2001). Para satisfacer estas demandas la glándula study was obtained on calving day $\left(7.27 \mathrm{mg}^{-\mathrm{dl}^{-1}}\right)$ and on average the cows exceed the threshold of subclinical hypocalcemia $\left(8.0 \mathrm{mg} . \mathrm{dl}^{-1}\right)$ until day 7 after calving. The blood calcium concentration wasn't statistically associated to reproductive indicators. Multiparous cows with calcium concentrations lower than $5.5 \mathrm{mg} \cdot \mathrm{dl}^{-1}$ on calving day had statistically $(\mathrm{p}<0.05)$ lower: daily milk, actual milk yield and lactation length compared to multiparous cows with blood calcium concentrations higher than or equal to $5.5 \mathrm{mg}^{\mathrm{ddl}}{ }^{-1}$. These findings indicate that blood calcium concentration during peripartum is an associated factor to yield performance in multiparous cows.

mamaria tiene que remover entre 20 y $30 \mathrm{~g}$ de Ca por día del plasma sanguíneo del animal, que causa un desbalance en el metabolismo del calcio (Horst 1984, Horst et al. 1994, Goff 2006).

En algunos casos, el sistema homeostático logra compensar la extracción de Ca de la glándula mamaria rápidamente, con lo cual hay una leve caída de la concentración de Ca sanguíneo, sin llegar a ser inferior a $8 \mathrm{mg} \cdot \mathrm{dl}^{-1}$. Esta situación se denomina normocalcemia y no llega a afectar la salud del animal. Cuando el animal no logra compensar esa caída en los niveles de Ca sanguíneo, se produce la hipocalcemia subclínica y puede ser diagnosticada mediante un análisis de sangre durante el periparto (Amaral 2014). Un animal bajo estas condiciones puede reducir la productividad al disminuir el consumo de materia seca al inicio de la lactancia.

Si la caída de Ca sanguíneo sobrepasa la capacidad del sistema homeostático para mantener la normocalcemia, la vaca presenta hipocalcemia clínica o fiebre de leche, que se caracteriza por que el animal sufre de inapetencia, tetania, inhibición de la micción y la defecación, decúbito lateral, y eventual coma y muerte si no se trata (Horst et al. 1997). Esta condición causa que el animal experimente cambios bioquímicos y 
fisiológicos que lo predisponen a enfermedades como mastitis, retención de placenta, desplazamiento de abomaso y cetosis (Chapinal et al. 2011, Horst et al. 2006, Roche y Berry 2006). Alrededor de un 5\% de los animales que sufren hipocalcemia clínica mueren (Goff 2006). Por lo menos un estudio reportó disminución de tratamientos por síntomas asociados a hipocalcemia posparto al realizar suplementación profiláctica de calcio inmediatamente después del parto, sin efectos positivos en la disminución de otras enfermedades asociadas al periparto (Miltenburg et al. 2016).

Martens y Kasebieter (1983) encontraron que las concentraciones altas de potasio en el líquido ruminal despolarizan la membrana apical de las papilas ruminales, que reducen el potencial electromotriz requerido para que el magnesio traspase la pared del rumen y sea metabolizado. El magnesio es indispensable para el buen funcionamiento del metabolismo del calcio (Goff 2008). Lo anterior hace que la nutrición preparto de la vaca lechera sea muy sensible en aquellos sistemas de producción que hacen un uso muy intensivo de las pasturas altas en potasio (superiores a 3\% de la materia seca), según Sánchez y Saborío-Montero (2014b).

Contenidos de potasio del orden de 3,5\% de la materia seca son comunes en los pastos suculentos del trópico (Sánchez 2008). El contenido de potasio en la materia seca de la dieta es utilizado para estimar la diferencia catiónanión dietética (DCAD), valores altos de potasio en la dieta incrementan la DCAD (DeGaris y Lean 2008). Un meta análisis (Lean et al. 2006) estableció que la DCAD es determinante en la ocurrencia de fiebre de leche, donde dietas con valores más altos de DCAD predisponen a las vacas a padecer la enfermedad. Otra investigación encontró que la absorción y excreción renal de magnesio en vacas lecheras incrementó al disminuir la DCAD (Roche et al. 2003).

La asociación entre la concentración de calcio en sangre de vacas lecheras durante el periparto e indicadores productivos y reproductivos no es concluyente entre estudios, existen investigaciones (Chapinal et al. 2012a, Chapinal et al. 2012b) que indican que bajas concentraciones de calcio cercanas al parto se asocian a disminución del desempeño productivo y reproductivo durante la lactación temprana. Por otra parte, otras investigaciones (Chamberlin et al. 2013) no indican una afectación de dichos desempeños en función a la concentración de este mineral en la sangre de vacas lecheras.

El objetivo de esta investigación fue describir la dinámica de la concentración de calcio en sangre durante el periparto del hato en estudio, para asociar las concentraciones de calcio con indicadores productivos y reproductivos. El conocimiento de la dinámica del calcio sanguíneo durante el periparto puede contribuir al desarrollo de prácticas de alimentación que favorezcan el desempeño óptimo de los animales durante el periodo periparto.

\section{MATERIALES Y MÉTODOS}

Durante el periodo comprendido entre enero y junio de 2014 se muestreó un total de 161 vacas Jersey en pastoreo en una finca ubicada en Oreamuno, Cartago, Costa Rica $\left(9^{\circ}\right.$ 55> Latitud Norte, $83^{\circ}$ 51> Longitud Oeste, 2100 a 2400 msnm). La alimentación de los animales se basó en el pastoreo intensivo de kikuyo (Kikuyuocloa clandestina) $\left(15,6 \%\right.$ de PC, 1,65 Mcal de $\mathrm{EN}_{\mathrm{L}}$ (3X), 0,35\% de Ca, 0,32\% de $\mathrm{Mg}, 3,5 \%$ de $\mathrm{K}$ ), y se suplementó con $4 \mathrm{~kg} \cdot \mathrm{vaca}^{-1}$.día ${ }^{-1}$ de una mezcla de granos baja en calcio (88\% MS, $14 \%$ PC, $0,2 \%$ $\mathrm{Ca}, 0,42 \% \mathrm{Mg}$ y $1,38 \% \mathrm{~K})$, y $1 \mathrm{~kg}$ de heno $(82,3 \%$ MS, 5,1\% PC, $0,4 \%$ Ca, 0,35\% Mg y 1,8\% K).día ${ }^{-1}$. Inmediatamente después del parto los animales fueron suplementados con $1 \mathrm{~kg}$ de una mezcla de granos $(87,5 \% \mathrm{MS}, 18,6 \% \mathrm{PC}, 0,90 \% \mathrm{Ca}, 0,42 \%$ $\mathrm{Mg}$ y $1,38 \% \mathrm{~K}$ ) por cada $2,5 \mathrm{~kg}$ de leche.

Se tomaron un total de 1072 muestras de sangre de los vasos sanguíneos coccígeos con agujas de 21" x 1" y tubos estériles de $9 \mathrm{ml}$ con activador de aglutinación de suero Z (Greiner Bio-One, tapa roja), desde 7 días antes del parto hasta 7 días posparto. Las muestras fueron identificadas y mantenidas en hielo mientras se 
trasladaron en un periodo inferior a 3 horas desde la finca hasta el Centro de Investigaciones en Nutrición Animal (CINA) de la Universidad de Costa Rica, donde se centrifugaron por $15 \mathrm{~min}$ a $4^{\circ} \mathrm{C}, 3500 \mathrm{rpm}$ y se separó el suero el mismo día del traslado desde la finca. El suero centrifugado fue conservado en congelación $\left(-20^{\circ} \mathrm{C}\right)$ hasta su análisis. Los contenidos de calcio en las muestras de suero fueron determinados por espectrofotometría de absorción atómica (Zettner y Seligson 1964), utilizando un espectrofotómetro Perkin Elmer AAnalyst 800, equipado con un auto muestreador automático S10. Un umbral de Ca sérico de menos de 2,0 mmol. $\mathrm{l}^{-1}\left(8 \mathrm{mg} \cdot \mathrm{dl}^{-1}\right)$, pero superior a $1,4 \mathrm{mmol}^{-\mathrm{l}^{-1}}\left(5,5 \mathrm{mg}^{\mathrm{d}} \mathrm{dl}^{-1}\right)$ se utilizó para definir a las vacas como positivas para hipocalcemia subclínica y un valor inferior a 1,4 mmol. $\mathrm{l}^{-1}\left(5,5 \mathrm{mg} . \mathrm{dl}^{-1}\right)$ para hipocalcemia clínica. Las muestras se clasificaron según parición de las vacas muestreadas en primíparas (animales de primer parto, $n=35$ ) y multíparas ( $\geq 2$ partos, $\mathrm{n}=126$ ), así como según los días en relación con el día de parición.

La información productiva, reproductiva y de concentración de calcio se registró en una base de datos en Excel $^{\circledR}$ y se analizó mediante los programas estadísticos IBM $^{\circledR}$ SPSS $^{\circledR}$ Statistics (versión 21; IBM Corp., Armonk, NY) e InfoStat 1.0 (Di Rienzo et al. 2011). Para la concentración de calcio se realizó un análisis de varianza (ANDEVA) de medidas repetidas con los efectos fijos de clase de parición (primípara o multípara) y vaca anidada dentro de parición como el sujeto de las medidas repetidas de tiempo. Asimismo, se utilizó la prueba t de Student para comparar los promedios de 2 categorías y una prueba de comparación de medias de Tukey para contrastar entre medias de 3 categorías. La significancia de las pruebas estadísticas se declaró cuando el valor $\mathrm{p}$ fue menor a 0,05 .

\section{RESULTADOS Y DISCUSIÓN}

La concentración de calcio promedio general disminuyó por debajo de $8,0 \mathrm{mg}^{-\mathrm{dl}^{-1}}$ a partir de 3 días antes del parto $\left(7,83 \mathrm{mg}^{-\mathrm{dl}^{-1}}\right)$ y hasta cuando parió la vaca $\left(7,27 \mathrm{mg}_{\mathrm{dll}}{ }^{-1}\right)$, día en que se obtuvo el valor mínimo de concentración de calcio (Figura 1). A partir del momento del parto, los niveles de calcio en sangre aumentaron hasta el día 3 posparto, donde hubo un periodo de

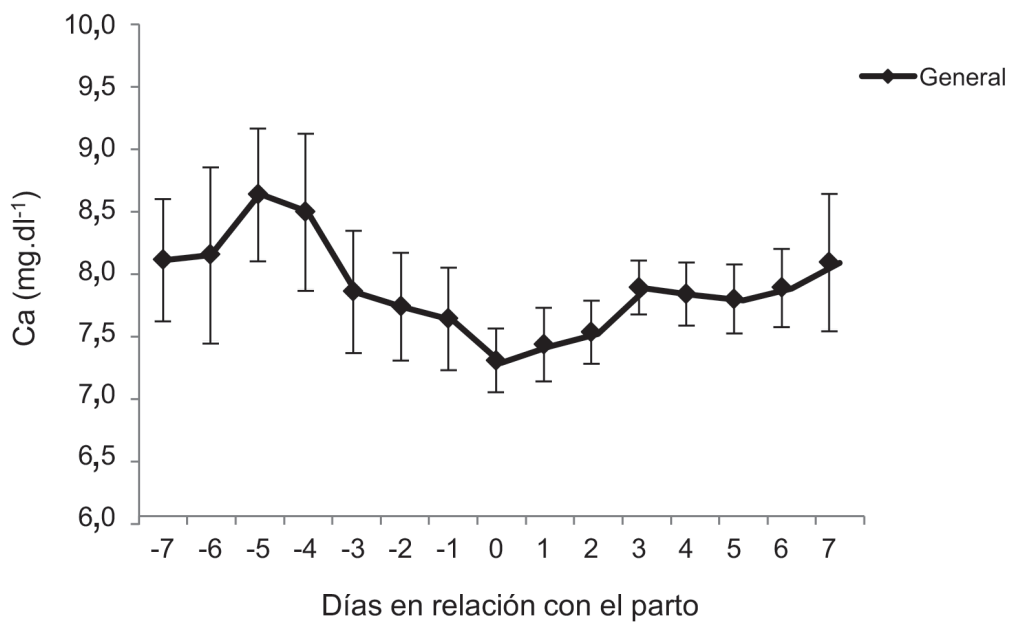

Fig. 1. Concentración de calcio en suero sanguíneo $\left(\mathrm{mg}^{\mathrm{d}} \mathrm{dl}^{-1}\right)$ en el periparto de vacas Jersey en pastoreo en Oreamuno, Cartago, Costa Rica según días en relación con el parto. 
4 días ( $3^{\circ}$ a $6^{\circ}$ posparto), en el cual se estabilizó dicha concentración en valores levemente inferiores al valor de normocalcemia $(7,86 ; 7,81 ; 7,77$ y $7,86 \mathrm{mg} \mathrm{dl}^{-1}$, respectivamente). El día 7 posparto se superó el valor umbral para normocalcemia (8,0 mg.dl-1) el cual llegó a 8,07 mg.dl- ${ }^{-1}$.

Resultados similares en relación con la concentración de calcio en sangre se encuentran en investigaciones en otras latitudes (Kimura et al. 2006, Chapinal et al. 2011, Chapinal et al. 2012a, Chamberlin et al. 2013), donde el día del parto es el momento de concentración mínima de calcio en sangre de los animales. Kimura et al. (2006) clasificaron a los animales según presencia o ausencia de fiebre de leche, en ese estudio los animales positivos a la enfermedad mostraron concentraciones promedio inferiores a $5 \mathrm{mg}^{-\mathrm{dl}^{-1}}$ de calcio en sangre al día del parto y recuperaron la normocalcemia el día 3 posparto. Esto sugiere que las vacas requieren de varios días para que la absorción de calcio a nivel intestinal y la resorción ósea respondan a la mayor demanda fisiológica de Ca (Goff y Horst 1997).

Al clasificar los animales según vacas primíparas y multíparas, se observaron diferencias en la concentración de calcio sanguíneo durante el periparto (Figura 2). En el caso de las vacas primíparas el promedio de calcio en sangre únicamente fue inferior al valor de normocalcemia $\left(8,0 \mathrm{mg} \cdot \mathrm{dl}^{-1}\right)$ el día del parto $\left(7,67 \mathrm{mg} \cdot \mathrm{dl}^{-1}\right)$

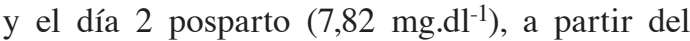
día 3 posparto y hasta el 7, las vacas primíparas alcanzaron concentraciones de calcio mayores a $8,0 \mathrm{mg} \cdot \mathrm{dl}^{-1}$.

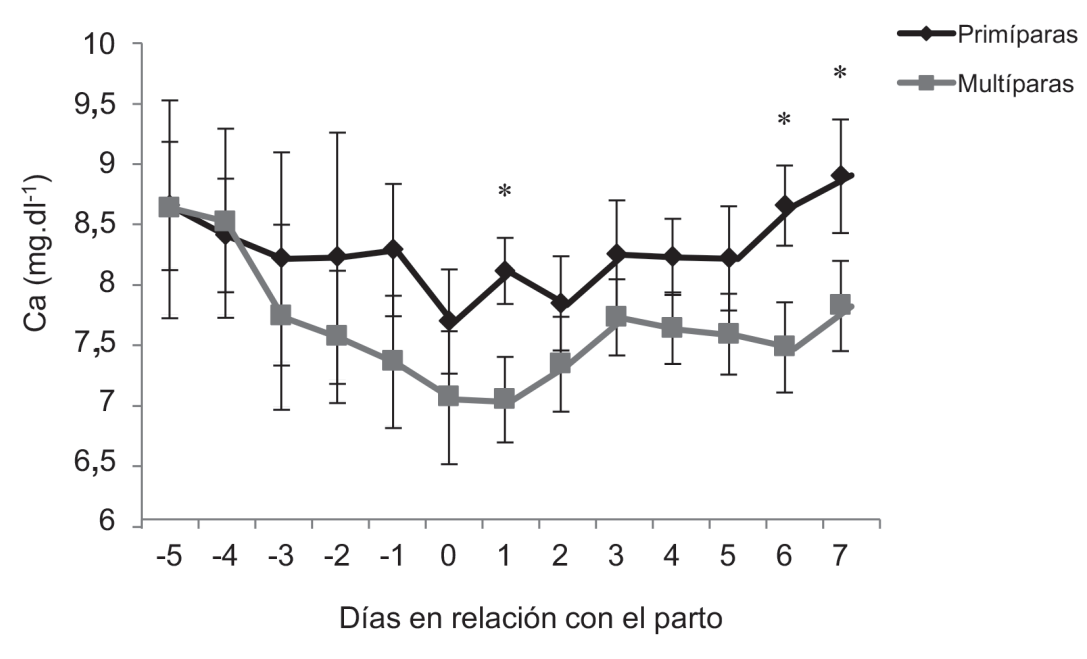

* Difieren significativamente $(\mathrm{p}<0,05)$ según la prueba t de Student.

Fig. 2. Concentración de calcio en suero sanguíneo en el periparto de vacas Jersey primíparas y multíparas en pastoreo en Oreamuno, Cartago, Costa Rica según días en relación con el parto.

En el caso de las vacas multíparas, los niveles de calcio en sangre decrecieron desde el

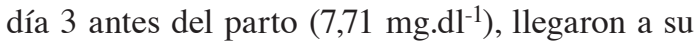
valor mínimo el día 1 posparto $\left(7,01 \mathrm{mg}^{\left.-\mathrm{dl}^{-1}\right)}\right.$ y se mantuvieron por debajo del nivel de normocalcemia, inclusive, hasta el 7 (7,80 $\left.\mathrm{mg}^{\mathrm{dl}} \mathrm{l}^{-1}\right)$. Reinhardt et al. (2011), Gild et al. (2015) y Sánchez y Saborío-Montero (2014a) muestran que los niveles de calcio en las vacas lecheras disminuyen conforme aumenta el número de lactancia; de igual forma, el NRC (2001) afirma que entre más partos tenga una vaca, el contenido de Ca sanguíneo al 
momento del parto es menor y la probabilidad de sufrir fiebre de leche aumenta.

El Cuadro 1 muestra la proporción de vacas primíparas, multíparas o general clasificadas como clínicas, subclínicas o sanas, según las muestras de suero sanguíneo con concentración mínima de calcio para cada animal en el periodo de 7 días preparto a 7 días posparto. En el Cuadro 1 se observa que la mayoría de las vacas primíparas $(69,2 \%)$ en algún día del periodo analizado presentaron una concentración de calcio en el rango de hipocalcemia subclínica. Una proporción más baja $(15,4 \%)$ de estas vacas presentaron valores dentro del ámbito de hipocalcemia clínica. En el caso de las vacas multíparas la proporción de animales clasificados como subclínicos fue 59,5\%, mientras que las vacas multíparas clasificadas como clínicas fueron $37,8 \%$. Estos resultados revelan que una gran proporción de las vacas, tanto primíparas como multíparas, tienen concentraciones de calcio en sangre durante el periparto por debajo de los umbrales considerados como óptimos y que las vacas multíparas son las que presentan niveles más bajos de este mineral y una mayor proporción de animales afectados.

Cuadro 1. Proporción de vacas primíparas, multíparas o general clasificadas como clínicas, subclínicas o sanas según las muestras de suero sanguíneo con concentración mínima de calcio para cada animal en el periodo de 7 días preparto a 7 días posparto en un hato Jersey en pastoreo en Oreamuno, Cartago, Costa Rica.

\begin{tabular}{lccc}
\hline & Clínicas & Subclínicas (\%) & Sanas \\
\hline Primíparas & 15,4 & 69,2 & 15,4 \\
Multíparas & 37,8 & 59,5 & 2,7 \\
General & 33,6 & 61,3 & 5,1 \\
\hline
\end{tabular}

En la literatura se reporta que la proporción de casos de hipocalcemia subclínica en ganado bovino lechero es mayor que la de hipocalcemia clínica, valores de 5\% de hipocalcemia clínica han sido asociados con incidencias de hipocalcemia subclínica del orden de 33\% (Roche 2003).

El número de parto es el factor de riesgo que tiene mayor efecto sobre la incidencia de hipocalcemia clínica, en donde un animal de 3 y 6 o más partos tiene respectivamente 11,8 (IC 95\% 9,12-15,27) y 52,4 (IC 95\% 40,9467,08 ) veces la probabilidad de sufrir fiebre de leche, en comparación con un animal de primer parto (Saborío-Montero et al. 2015). Esto coincide con los hallazgos de Sánchez y SaboríoMontero (2014a, 2014b) quienes encontraron, en vacas estudiadas en varias fincas de la zona de Cartago, Costa Rica, un patrón similar en cuanto al aumento de la incidencia de fiebre de leche conforme avanzaba el número de lactancia.
Un estudio (Goff et al. 1995) argumentó que el incremento en susceptibilidad a fiebre de leche de las vacas adultas podría ser el resultado de menos receptores para $1,25(\mathrm{OH})_{2} \mathrm{D}_{3}$ en el intestino en comparación con animales jóvenes.

En relación con los indicadores productivos, se observaron algunas diferencias al analizar las medias de dichos indicadores según la concentración de calcio sanguíneo en el periparto de las vacas primíparas y multíparas, no así para los indicadores reproductivos (Cuadro 2).

La producción de leche corregida a 305 días en las vacas primíparas fue mayor $(\mathrm{p}<0,05)$ para los animales con concentraciones de calcio entre 5,5 y $8,0 \mathrm{mg} . \mathrm{dl}^{-1}$ el día previo al parto, en relación con las vacas primíparas con concentraciones de calcio superiores a $8,0 \mathrm{mg}_{\mathrm{dl}}{ }^{-1}$ para ese mismo día (Cuadro 2). Estos resultados indican que la asociación entre indicadores productivos y concentración de calcio sanguíneo durante el periparto podría ser 


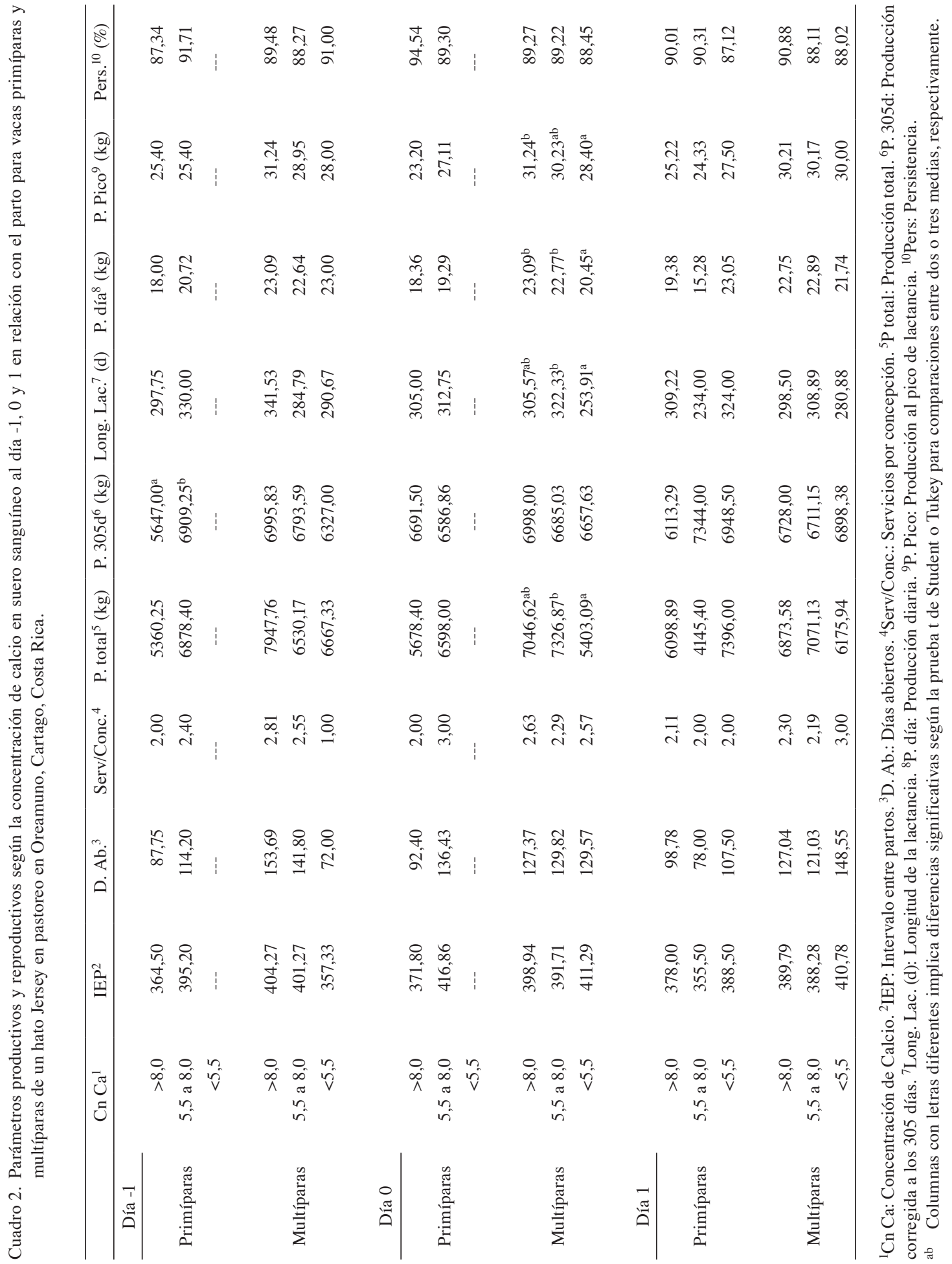


distinta para animales de primer parto en relación con animales con más de un parto.

Algunas investigaciones establecen que la producción de leche en vacas primíparas no responde de igual manera a un mismo manejo alimenticio en comparación con vacas multíparas durante el periparto (DeGroot et al. 2010) y que las vacas primíparas podrían beneficiarse de un manejo distinto al de las multíparas en el periodo de transición (Neave et al. 2017). Otros estudios también han reportado mayores producciones de leche para vacas con hipocalcemia subclínica en relación con animales sanos (Jawor et al. 2012).

Las vacas multíparas con concentraciones de calcio inferiores a 5,5 mg. $\mathrm{dl}^{-1}$ el día del parto tuvieron estadísticamente $(\mathrm{p}<0,05)$ menor: producción por día, producción total y longitud de lactancia, en relación con vacas multíparas con concentraciones de calcio mayor o igual a $5,5 \mathrm{mg} \cdot \mathrm{dl}^{-1}$. El pico de producción de leche fue menor $(\mathrm{p}<0,05)$ para las vacas multíparas con

A

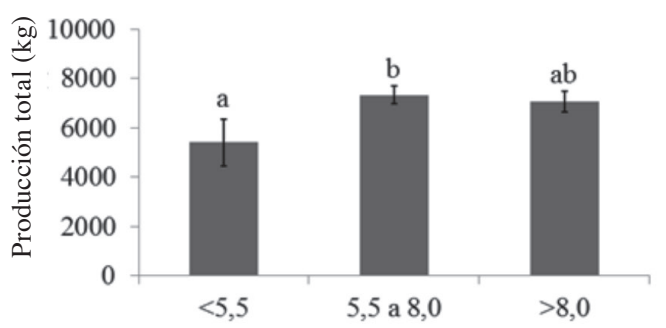

C Concentración de Ca mg. $\mathrm{dl}^{-1}$

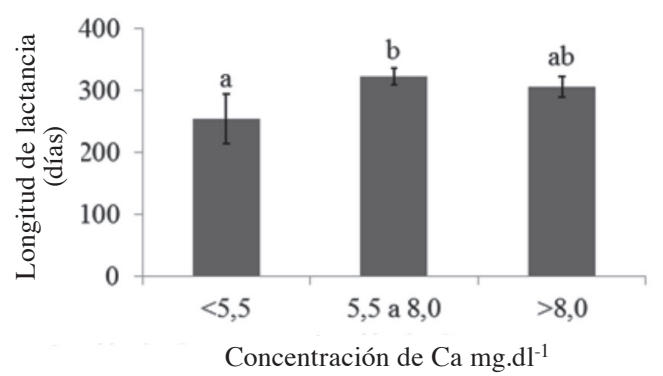

concentraciones de calcio inferiores a $5,5 \mathrm{mg}^{-\mathrm{dl}^{-1}}$ $(28,40 \mathrm{~kg})$ en relación con animales cuya concentración de calcio fue superior a $8,0 \mathrm{mg}^{-d l^{-1}}(31,24$ $\mathrm{kg}$ ) el día del parto.

Según Block (1993) y Weich et al. (2013), una dieta aniónica, en el periodo preparto, ayuda a disminuir la incidencia de hipocalcemia y a la vez aumenta la producción de leche en esa lactancia. De igual forma, otras investigaciones (Dann et al. 2005, Dohoo y Martin 1984) han reportado que los animales con fiebre de leche tuvieron menores rendimientos de producción láctea. Chapinal et al. (2012a), mencionan que a mayor proporción de animales con hipocalcemia en el periparto, mayor riesgo de una menor producción de leche en las primeras mediciones posparto.

La producción total, producción diaria, pico de producción de leche y la longitud de la lactancia fue menor para las vacas multíparas con concentraciones de calcio inferiores a 5,5 mg.dl-1 el día del parto (Figura 3).

\section{B}
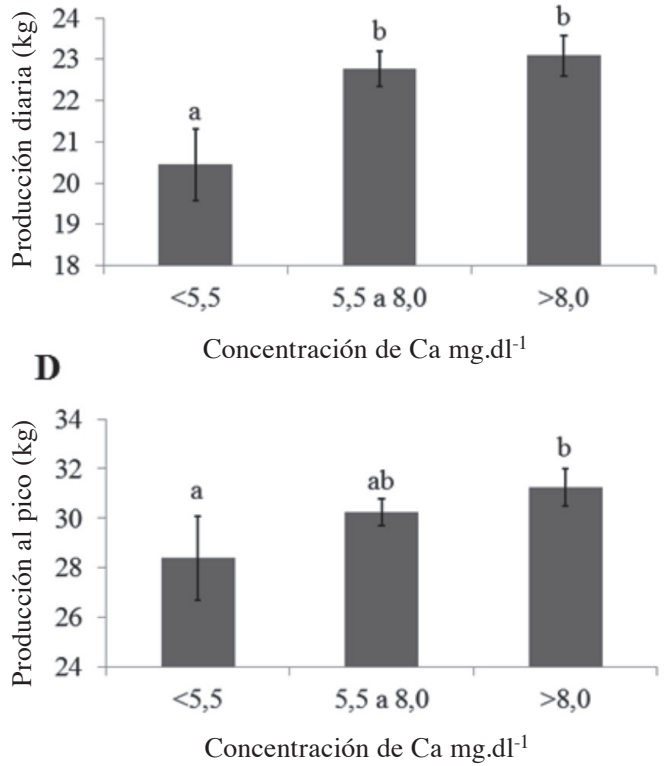

${ }^{\text {ab }}$ Columnas con letras diferentes implica diferencias significativas según la prueba de Tukey.

Fig. 3. Producción total (A), producción diaria (B), longitud de lactancia (C) y producción al pico (D) de vacas multíparas según la concentración de calcio en el suero sanguíneo al día del parto en un hato Jersey en pastoreo en Oreamuno, Cartago, Costa Rica. 
Estos resultados demuestran que existe una asociación, no necesariamente causal entre la concentración sanguínea de calcio y el desempeño productivo de las vacas multíparas, lo que plantea la posibilidad de que cambios dirigidos a una mejora en las medidas profilácticas que incrementen la concentración de calcio el día del parto podría generar una mejora en algunos indicadores de producción de interés para el sector lechero.

\section{CONCLUSIONES}

La dinámica de la concentración de calcio durante el periparto de las vacas estudiadas fue similar a lo encontrado en la literatura, donde la concentración de calcio disminuye marcadamente en los días más cercanos al parto. Se observó que la homeostasis del calcio en sangre durante el periparto fue menos eficiente en vacas multíparas que en primíparas. Varios parámetros productivos de interés fueron asociados a la concentración de calcio en el día del parto en vacas multíparas, con lo cual se identificó que los animales que se mantienen más cercanos a la normocalcemia logran mejores producciones de leche.

\section{AGRADECIMIENTOS}

Los autores expresan su agradecimiento a los propietarios de la finca "El Plantón", Sr. Julio Sancho y Sr. Álvaro Sancho, por facilitar el hato de ganado lechero donde se realizó este estudio, además, al Sr. Johnny Calderón Chacón y a los demás colaboradores de la finca por su oportuna ayuda.

\section{LITERATURA CITADA}

Amaral, D. 2014. Subclinical hypocalcemia, or milk fever, in dairy cows-why all the fuss. University of Kentucky (en línea). Consultado 26 abr. 2017. Disponible en http://www.extension.org/pages/70227/subclinicalhypocalcemia-or-milk-fever-in-dairy-cows-why-allthe-fuss\#.U159C6Iyv7c

Australia, State Government of Victoria. 2007. Department of Envionmental and Primary Industries. Agriculture and Food. Milk fever (Hypocalcemia) in cows (en línea). Consultado 30 dic. 2016. Disponible en http://www.depi.vic.gov.au/agriculture-and-food/ pests-diseases-and-weeds/animal-diseases/beefand-dairy-cows/milk-fever-hypocalcaemia-in-cows

Block, E. 1993. Manipulation of dietary cation-anion difference on nutritionally related production diseases, productivity, and metabolic responses of dairy cows. J. Dairy Sci. 77:1437-1450.

Chamberlin, WG; Middleton, JR; Spain, JN; Johnson, GC; Ellersieck, MR; Pithua, P. 2013. Subclinical hypocalcemia, plasma biochemical parameters, lipid metabolism, postpartum disease, and fertility in postparturient dairy cows. J. Dairy Sci. 96:7001-7013.

Chapinal, N; Carson, M; Duffield, TF; Capel, M; Godden, S; Overton, M; Santos, JEP; Leblanc, SJ. 2011. The association of serum metabolites with clinical disease during the transition period. J. Dairy Sci. 94:4897-4903.

Chapinal, N; Carson, ME; Leblanc, SJ; Leslie, KE; Godden, S; Capel, M; Santos, JEP; Overton, MW; Duffield, TF. 2012a. The association of serum metabolites in the transition period with milk production and early lactation reproductive performance. J. Dairy Sci. 95:1301-1309.

Chapinal, N; Leblanc, SJ; Carson, ME; Leslie, KE; Godden, S; Capel, M; Santos, JEP; Overton, MW; Duffield, TF. 2012b. Herd-level association of serum metabolites in the transition period with disease, milk production, and early lactation reproductive performance. J. Dairy Sci. 95:5676-5682.

Dann, HM; Morin, DE; Bollero, GA; Murphy, MR Drackley JK. 2005. Prepartum intake, postpartum induction of ketosis, and periparturient disorders affect the metabolic status of dairy cows. J. Dairy Sci. 88:3249-3264.

DeGaris, PJ; Lean, IJ. 2008. Milk fever in dairy cows: A review of pathophysiology and control principles. The Veterinary Journal 176(1):58-69.

DeGroot, MA; Block, E; French, PD. 2010. Effect of prepartum anionic supplementation on periparturient feed intake, health and milk production. J. Dairy Sci. 93:5268-5279.

Di Rienzo, JA; Casanoves, F; Balzarini, MG; Gonzalez, L; Tablada M; Robledo, CW. 2011. InfoStat versión 2011. Grupo InfoStat, FCA (en línea, programa informático). Córdoba, Argentina, Universidad Nacional de Córdoba. Consultado 10 jun. 2016. Disponible en http://www.infostat.com.ar

Dohoo, IR; Martin, SW. 1984. Disease, production and culling in Holstein-Friesian cows. III. Disease and production as determinants of disease. Preventive Veterinary Medicine 2:671-690.

Gild, C; Alpert, N; Straten, M. 2015. The influence of sublinical hypocalcemia on production and 
reproduction parameters in Israeli dairy herds. Israel Journal of Veterinary Medicine 70(1):16-21.

Goff, JP. 2006. Macromineral physiology and application to the feeding of the dairy cow for prevention of milk fever and other periparturient mineral disorders. Animal Feed Science and Technology 126:237-257.

Goff, JP. 2008. The monitoring, prevention and treatment of milk fever and subclinical hypocalcemia in dairy cows. Vet. J. 176:50-57.

Goff, JP; Horst, RL. 1997. Physiological changes at parturition and their relationship to metabolic disorders. J. Dairy Sci. 80:1260-1268.

Goff, JP; Reinhardt, T; Horst, RL. 1995. Milk fever and dietary cation-anion balance effects on concentration of vitamin D receptor in tissue of periparturient dairy cows. J. Dairy Sci. 78:2388-2394.

Horst, RL. 1984. Regulation of calcium and phosphorus homeostasis in the dairy cow. J. Dairy Sci. 69:604-616.

Horts, RL; Goff, JP; McCluskey, BJ. 2006. Prevalence of subclinical hypocalcemia in U.S. dairy operations. In Joshi $\mathrm{N}$; Herdt $\mathrm{TH}$ (eds.). Production diseases in farm animals. Wageningen, The Netherlands, Wageningen Academic Publishers. p. 215.

Horst, RL; Goff, JP; Reinhardt, TA. 1994. Calcium and vitamin D metabolism in the dairy cow. J. Dairy Sci. 77:1936-1951.

Horts, RL; Goff, JP; Reinhardt, TA; Buxton DR. 1997. Strategies for preventing milk fever in dairy cattle. J. Dairy Sci. 80:1269-1280.

Jawor P; Huzzey, JM; Leblanc, SJ; von Keyserlingk, MAG. 2012. Associations of subclinical hypocalcemia at calving with milk yield, and feeding, drinking, and standing behaviors around parturition in Holstein cows. J. Dairy Sci. 95:1240-1248.

Kimura, K; Reinhard, TA; Goff, JP. 2006. Parturition and hypocalcemia blunts calcium signals in immune cells of dairy cattle. J. Dairy Sci. 89:2588-2595.

Lean, IJ; Degaris, PJ; Mcneil, DM; Block, E. 2006. Hypocalcemia in dairy cows meta-analysis and dietary cation anion difference theory revisited. J. dairy Sci. 89(2):669-684.

Martens, H; Kasebieter, H. 1983. In vitro studies of the effect of sodium and potassium ions in magnesium transport across the isolated rumen mucosa of sheep. Zentralbl Veterinarmed (A) 30:1-14

Miltenburg, CL; Duffield, TF; Bienzle, D; Scholtz, EL; Leblanc, SJ. 2016. Randomized clinical trial of a calcium supplement for improvement of health in dairy cows in early lactation. J. Dairy Sci. 99(8):6550-6562.
NRC (National Research Council). 2001. Nutrient Requirements of Dairy Cattle. 7th rev. ed. National Academy Press. Washington, D.C. 381 p.

Neave, HW; Lomb, J; von Keyserlingk, MAG; BehnamShabahang, A; Weary, DM. 2017. Parity differences in the behavior of transition dairy cows. J. Dairy Sci. 100:548-561.

Reinhardt, TA; Lippolis, JD; McCluskey, BJ; Goff, JP; Horst, RL. 2011. Prevalence of subclinical hypocalcemia in dairy herds. The Veterinary Journal 188:122-124.

Roche, JR. 2003. The incidence and control of hypocalcemia in pasture-based systems. Acta Vet. Scand. Suppl. 97:141-144.

Roche, JR; Berry, DP. 2006. Periparturient climatic, animal, and management factors influencing the incidence of milk fever in grazing systems. J. Dairy Sci. 89:2775-2783.

Roche, JR; Dalley, D; Moate, P; Grainger, C; Rath, M; O'Mara, F. 2003. Dietary cation-anion difference and the health and production of pasture-fed dairy cows 2. Non-lactating periparturient cows. J. Dairy Sci. 86:979-987.

Saborío-Montero, A; Vargas, B; Romero, J; Sánchez; JM. 2015. Risk factors associated with milk fever occurrence in Costa Rican dairy cattle. J. Dairy Sci. 93:319-320.

Sánchez, JM. 2008. El pasto kikuyo y su aporte a la nutrición de vacas lecheras. In VI Seminario Internacional. Competitividad en carne y leche. Medellín, Colombia. p. 137-155.

Sánchez, JM; Goff, JP. 2006. Strategies for controlling hypocalcemia in dairy cows in confinement and pastures. In Joshi, N; Herdt TH (eds). Production diseases in farm animals. Wageningen, The Netherlands, Wageningen Academic Publishers. p. 182-187.

Sánchez, JM; Saborío-Montero, A. 2014a. Hipocalcemia e Hipomagnesemia en un hato de vacas Holstein, Jersey y Guernsey en pastoreo. Agronomía Costarricense 38(2):55-65.

Sánchez, JM; Saborío-Montero, A. 2014b. Prevalencia de hipocalcemia en cuatro hatos Jersey en pastoreo en Costa Rica. Agronomía Costarricense 38(2):33-41.

Weich, W; Block, E; Litherland, NB. 2013. Extended negative dietary cation-anion difference feeding does not negatively affect postpartum performance of multiparous Dairy cows. J. Dairy Sci. 96:5780-5792.

Zettner, A; Seligson, D. 1964. Application of atomic absorption spectrophotometry in the determination of calcium serum. Clin Chem. 10:869-890. 